\title{
ENGINEERING QUANTUM UNIVERSAL LOGIC GATES IN ELECTROMAGNETIC-FIELD MODES
}

\author{
Farhan Saif, Rameez ul Islam, and Mazhar Javed \\ Department of Electronics, Quaid-i-Azam University \\ 45320 Islamabad, Pakistan \\ e-mail: saif@fulbrightweb.org
}

\begin{abstract}
We present a study of quantum computing by engineering nonlocal quantum universal gates. The universal quantum logic gate is based on the interaction of a two-level atom with two modes of the electromagnetic field in a high $Q$ superconducting cavity. The two-level atom acts as a control qubit and the two-mode electromagnetic field serves as a target qubit. Presently available QED experiments make it possible to realize the theoretical suggestion in the laboratory. We check the feasibility of our proposal by calculating the success probability.
\end{abstract}

Keywords: quantum information, matter-field interaction, success probability.

\section{Introduction}

Application of principles of quantum mechanics in the development of computational techniques has given birth to the subject of quantum computation [1-3]. The reliable case of preparing and detecting quantum states, the scalability to a large number of qubits, the engineering of quantum entanglement, and the long decoherence time are the prime characteristics associated with a physical system to develop a practical quantum computer [4-6]. Keeping these objectives in view, numerous physical systems have been suggested to develop fast and reliable practical quantum computers, which include nuclear magnetic resonances (NMR) [7, 8], ion traps [9-11], quantum dots [12], quantum cavities [13-15], flying atoms [16, 17], photonic-crystal waveguides [18], and electron spins controlled by ultrafast off-resonant single optical pulses [19]. In quantum computers, operations are performed by means of single-qubit and multiple-qubit quantum logic gates [4-6] which lead to the generation of quantum-mechanical states in the system. The quantum universal logic gate comprises any two-qubit quantum gate together with a single-qubit Hadamard gate [20-23]. In addition, a quantum-phase gate also serves as a quantum universal logic gate $[24,25]$. There are various theoretical and experimental suggestions to implement a quantum-controlled NOT gate, which include Ramsey atomic interferometry, selective driving of optical resonances of two qubits undergoing a dipole-dipole interaction, and the Bragg scattering of atoms. In this paper, we engineer quantum universal logic gates by the controlled interaction of a circular Rydberg atom with a high $Q$ superconducting cavity in the electromagnetic-field modes. The atom is in resonance with the two modes in the presence and absence of a Stark field. Thus the Rydberg atom acts as a control qubit, whereas the two-mode elecrtomagnetic field provides a target qubit. Theoretical [26, 27] and experimental [28] schemes have been developed which lead to the entanglement in two modes of the

Manuscript submitted by the author in English on 21 September 2007.

1573-8760/07/2805-0529 @ ${ }^{\circ} 2007$ Springer Science+Business Media, Inc. 
electromagnetic field and thus demonstrate the case of preparing and detecting quantum qubit states. Here we show that universal quantum computing is feasible in this system.

The outline of the paper is as follows.

In Sec. 2 we suggest our model of the system, which was experimentally realized in [28]. In Secs. 3 and 4, respectively, we mention the quantum CNOT logic gate and a single-bit Hadamard gate. In Sec. 5 we summarize our results and discuss the success probability.

\section{The Model}

We consider a two-level circular Rydberg atom, which passes through a high $Q$ superconducting cavity. The cavity contains nondegenerate orthogonally polarized modes $M_{A}$ and $M_{B}$ with mode frequencies $\omega_{A}$ and $\omega_{B}$ (presented in Fig. 1). We consider the atomic transition frequency $\omega_{e g}=\omega_{e}-\omega_{g}$ in resonance with the field frequency $\omega_{A}$, where $\omega_{e}$ and $\omega_{g}$ are the frequencies associated with the excited state $|e\rangle$ and the ground state $|g\rangle$, respectively. In the presence of an electric field, the excited state $|e\rangle$ changes to the state $\left|e^{\prime}\right\rangle$, and the atomic transition frequency $\omega_{e^{\prime} g}$ becomes equal to the electromagnetic-field frequency $\omega_{B}$ due to the Stark effect [29].

Thus the atom emits a photon coherently in the cavity mode $M_{B}$ at a different frequency $\omega_{B}$ in the presence of the Stark field. Latter, the final state of the atom is analyzed in a state-selective fieldionization detector. We show that by controlling the coherent interaction it is possible to realize the two-qubit quantum CNOT logic gate and a single Hadamard gate in the system.

In order to realize our scheme in the laboratory, we follow the experimental setup of [28]. As a control bit, we consider a circular Rydberg rubidium atom, with principal quantum numbers 51 and 50 acting as levels $|e\rangle$ and $|g\rangle$, respectively. The transition from level $|e\rangle$ to level $|g\rangle$ occurs at frequency

|e)

$|\mathrm{g}\rangle$

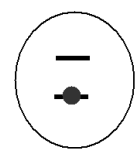

Atom

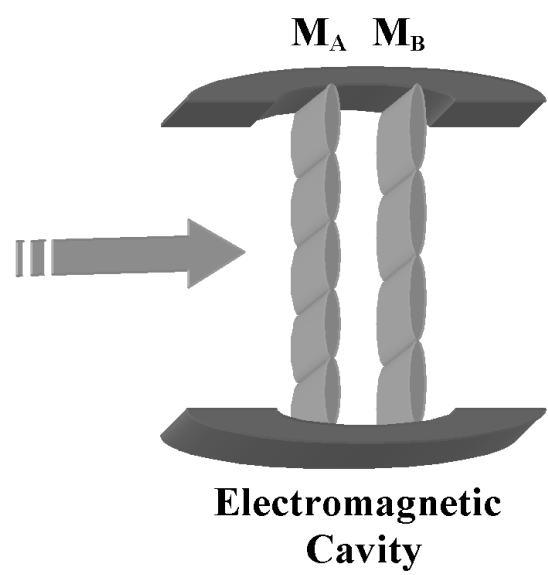

Fig. 1. Schematic diagram of the proposed experimental setup. $51.1 \mathrm{GHz}$. A very small injection rate makes the probability of having two atoms at the same time in the cavity very small. The optical cavity is a FabryPerot resonator made up of two spherical niobium mirrors. The two orthogonally polarized TEM 900 modes $\left(M_{A}\right.$ and $\left.M_{B}\right)$ have the same Gaussian geometry with waist $6 \mathrm{~mm}$. The frequency splitting occurs due to a slight mirror-shape anisotropy. The photon damping times are $T_{r, a}=1 \mathrm{~ms}$ and $T_{r, b}=0.9 \mathrm{~ms}$ for the electromagnetic-field modes $M_{A}$ and $M_{B}$, respectively.

\section{QED-Based CNOT Logic Gate}

The quantum-controlled NOT logic gate is a two-input-two-output logic gate which requires a control qubit $\left|q_{1}\right\rangle$ and a target qubit $\left|q_{2}\right\rangle$. The state of the control qubit $\left|q_{1}\right\rangle$ controls the state of the target qubit $\left|q_{2}\right\rangle$ such that

$$
\left|q_{1}\right\rangle\left|q_{2}\right\rangle \rightarrow\left|q_{1}\right\rangle\left|q_{1} \oplus q_{2}\right\rangle
$$


where $\oplus$ indicates addition modulus 2 . This implies that the target qubit is flipped if the control qubit carries logic one, and remains unchanged if the control qubit carries logic zero.

As discussed above, in our study we take the control qubit as a two-level atom, which is defined in a two-dimensional Hilbert space with $|e\rangle$ and $|g\rangle$ as basis vectors, where $|e\rangle$ expresses the excited state of the two-level atom and $|g\rangle$ indicates the ground state. The two nondegerate and orthogonally polarized cavity modes $M_{A}$ and $M_{B}$ make the target qubit $\left|q_{2}\right\rangle$. The target qubit is defined in the two-dimensional Hilbert space spanned by the state vector $\left|\chi_{1}\right\rangle=\left|1_{A}, 0_{B}\right\rangle$, which expresses the presence of one photon in mode $A$ when there is no photon in mode $B$, and the state vector $\left|\chi_{2}\right\rangle=\left|0_{A}, 1_{B}\right\rangle$, which indicates that the mode $A$ is in the vacuum state when one photon is present in the mode $B$.

The interaction of the two-level atom (acting as a control qubit) with the electromagnetic cavity containing two-field modes (acting as a target qubit) leads to the universal two-qubit control NOT logic gate and single-bit Hadamard gate. We prepare the two-level atom in the ground state $|g\rangle$ in a Ramsey cavity. It enters an electromagnetic cavity which contains a single photon of the field mode $M_{A}$, whereas mode $M_{B}$ is in the vacuum state. The transition frequency of the atom is taken equal to the frequency of mode $M_{A}$, hence, the atom interacts resonantly with the field.

The interaction of the two-level atom with the electromagnetic-field mode $M_{A}$ is described by the Jaynes-Cummings-Paul interaction Hamiltonian [29, 30] expressed as follows:

$$
\hat{V}=\hbar \mu_{1}\left(\hat{a}^{\dagger} \hat{\sigma}+\hat{\sigma}^{\dagger} \hat{a}\right)
$$

where $\hat{a}^{\dagger}(\hat{a})$ is the field creation (annihilation) operator, $\hat{\sigma}^{\dagger}=|e\rangle\langle g| \quad(\hat{\sigma}=|g\rangle\langle e|)$ is the atomic raising (lowering) operator, and $\mu_{1}$ is the coupling constant. Hence the atom-field combined state of the system becomes

$$
|\psi(t)\rangle=c_{g}(t)\left|g, 1_{A}\right\rangle+c_{e}(t)\left|e, 0_{A}\right\rangle .
$$

The probability amplitudes $c_{g}$ and $c_{e}$ govern the evolution of the atom initially in the ground state and change as a function of interaction time $t$ such that

$$
c_{g}(t)=\cos \left(\Omega_{A} t / 2\right), \quad c_{e}(t)=-i \sin \left(\Omega_{A} t / 2\right) .
$$

The frequency $\Omega_{A}=2 \mu_{1} \sqrt{n_{A}}$ describes the Rabi frequency of the atom in the mode $M_{A}$ containing $n_{A}$ number of photons. The atom interacts for a time $\pi / \Omega_{A}$ with mode $M_{A}$ and completes half of the Rabi oscillation. As a result, it absorbs the cavity photon in mode $M_{A}$ and jumps to the excited state $|e\rangle$.

After the interaction time $\pi / \Omega_{A}$, we apply a Stark field, which shifts the excited state from $|e\rangle$ to $|e ́\rangle$. Hence the atom observes a change in the transition energy and finds itself resonant with mode $M_{B}$. The atom interacts with the cavity mode $M_{B}$ resonantly and follows the interaction Hamiltonian, viz.,

$$
\hat{V}=\hbar \mu_{2}\left(\hat{b}^{\dagger} \hat{\sigma}^{\prime}+\hat{\sigma}^{\prime \dagger} \hat{b}\right)
$$

where $\hat{b}^{\dagger}(\hat{b})$ is the creation (annihilation) operator of the field mode $M_{B}, \hat{\sigma}^{\prime \dagger}=\left|e^{\prime}\right\rangle\langle g| \quad\left(\hat{\sigma}^{\prime}=|g\rangle\left\langle e^{\prime}\right|\right)$ is the atomic raising (lowering) operator, and $\mu_{2}$ is the coupling constant. The probability amplitudes of the resonant atom flips are $c_{e^{\prime}}(t)=\cos \left[\Omega_{B}\left(t-t_{0}\right) / 2\right]$ and $c_{g}(t)=-i \sin \left[\Omega_{B}\left(t-t_{0}\right) / 2\right]$, where $t>t_{0}=\pi / \Omega_{A}$ and $\Omega_{B}=2 \mu_{2} \sqrt{n_{B}+1}$, with $n_{B}$ describing the number of photons in cavity $B$. After interaction with mode $M_{B}$ for a time $\pi\left(\Omega_{A}+\Omega_{B}\right) / \Omega_{A} \Omega_{B}$, the atom leaves the cavity in the ground state $|g\rangle$ and thus contributes one photon to the cavity mode $M_{B}$. Therefore, the atom performs a swapping of electromagnetic fields between two field modes by a controlled interaction. 
In this case, we prepare the two-level atom in the ground state $|g\rangle$, where the electromagnetic cavity contains mode $M_{A}$ in the vacuum state and a single photon in the electromagnetic-field mode $M_{B}$. The atom becomes resonant with the electromagnetic-cavity-field mode $M_{B}$ in the presence of the Stark field at time $t=0$. It exhibits a controlled interaction with the field mode $M_{B}$ for a time $\pi / \Omega_{B}$ equal to half of the Rabi oscillation time, where $\Omega_{B}=2 \mu_{2} \sqrt{n_{B}}$. Thus the state of the system at $t=\pi / \Omega_{B}$ becomes $\left|e^{\prime}, 0,0\right\rangle$. We switch off the Stark field and let the atom interact resonantly with mode $M_{A}$ for a time $\pi\left(\Omega_{A}+\Omega_{B}\right) / \Omega_{A} \Omega_{B}$, where $\Omega_{A}=2 \mu_{1} \sqrt{n_{A}+1}$. Therefore, the atom again leaves the cavity in the ground state $|g\rangle$ and performs field swapping by contributing one photon to the field mode $M_{A}$.

The target qubit made up of the electromagnetic fields remains unchanged if the control qubit, that is, the two-level atom, is initially in the excited state. The atom prepared in the excited state $|e\rangle$ interacts with mode $M_{A}$ containing one-photon field. The Rabi oscillation frequency is given by $\Omega_{A}=2 \mu_{1} \sqrt{n_{A}+1}$; thus it completes one Rabi oscillation in time $2 \pi / \Omega_{A}=\sqrt{2} \pi / \mu_{1}$. The atom leaves the cavity in state $|e\rangle$ without disturbing the state of the cavity-field modes.

If the cavity-field mode $M_{B}$ is in the Fock state $|1\rangle$, the atom becomes resonant with $M_{B}$ in the presence of a Stark field to introduce zero detunning between the atomic-transition frequency and the field frequency. Hence, the atom completes one Rabi oscillation in the field for the interaction time $\sqrt{2} \pi / \mu_{2}$ and leaves the cavity in the excited state $|e\rangle$ without contributing to the radiation-field mode $M_{B}$.

\section{Single Qubit Hadamard Logic Gate}

The Hadamard gate generates the superposition state of a qubit provided the system is in one of the basis vectors of the two-dimensional Hilbert space. Hence, we consider the control qubit in state $|g\rangle$ and target qubit in the electromagnetic field state $\left|\chi_{1}\right\rangle=\left|1_{A}, 0_{B}\right\rangle$. The atom interacts with the cavity for a time equal to one-fourth of the Rabi-oscillation period, that is, $\pi / 2 \Omega_{A}$. Thus the combined atom-field state of the system becomes $\left(\left|e, 0_{A}, 0_{B}\right\rangle+\left|g, 1_{A}, 0_{B}\right\rangle\right) / \sqrt{2}$. Later, we introduce a Stark field, which shifts the excited state from $|e\rangle$ to $\left|e^{\prime}\right\rangle$. Thus the atom interacts in resonance with mode $M_{B}$. The atom leaves the cavity after time $\pi / \Omega_{B}$ in the ground state $|g\rangle$ and contributes one photon to the field in mode $M_{B}$. The resultant state of the system becomes a superposition state of $\left|\chi_{1}\right\rangle$ and $\left|\chi_{2}\right\rangle$, with an equal probability to find the system in either of the two basis vectors.

\section{Discussions}

In this paper, we present a scheme to engineer the two-qubit quantum-controlled NOT logic gate and a single-bit Hadamard gate by a controlled interaction between two-mode high $Q$ electromagnetic-cavity field and a two-level atom. For this purpose, we take the two-level atom as the control qubit, whereas the target qubit is made up of two modes of the cavity field.

The proposed set of universal logic gates can operate at high enough success probabilities with ideal unit fidelities. Success probabilities depend mainly upon the precise selection of the interaction times of the two-level atom with the successive cavity modes. Imprecision in the interaction times occur mainly due to the initial velocity spread of the two-level atom, with minor contributions coming from factors 
like coupling dispersion. In this respect, the success probability for the CNOT gate is

$$
P_{s}=1-\left[\sin ^{2}\left(\frac{\Omega_{A} \Delta t_{A}}{2}\right)+\cos ^{2}\left(\frac{\Omega_{A} \Delta t_{A}}{2}\right) \sin ^{2}\left(\frac{\Omega_{B} \Delta t_{B}}{2}\right)\right],
$$

where $\Delta t_{A}$ and $\Delta t_{B}$ stand for the uncontrollable imprecision in the interaction times of the atom with the field modes $M_{A}$ and $M_{B}$, respectively. A plot of the CNOT-gate success probability versus $\Delta t_{A}$ and $\Delta t_{B}$ is shown in Fig. 2. Now, for the atoms generated out of a thermal source, i.e., an oven, a velocity spread of $\pm 2 \mathrm{~m} / \mathrm{s}$ has been reported when the average velocity is taken around about $503 \mathrm{~m} / \mathrm{s}$ [28]. However, a much better control over atomic velocities is now available through magneto-optical trapping and cooling of neutral atoms [31,32]. Therefore, under the present cavity QED research scenario, the quantum logic gates proposed are expected to produce results with excellent success probabilities.

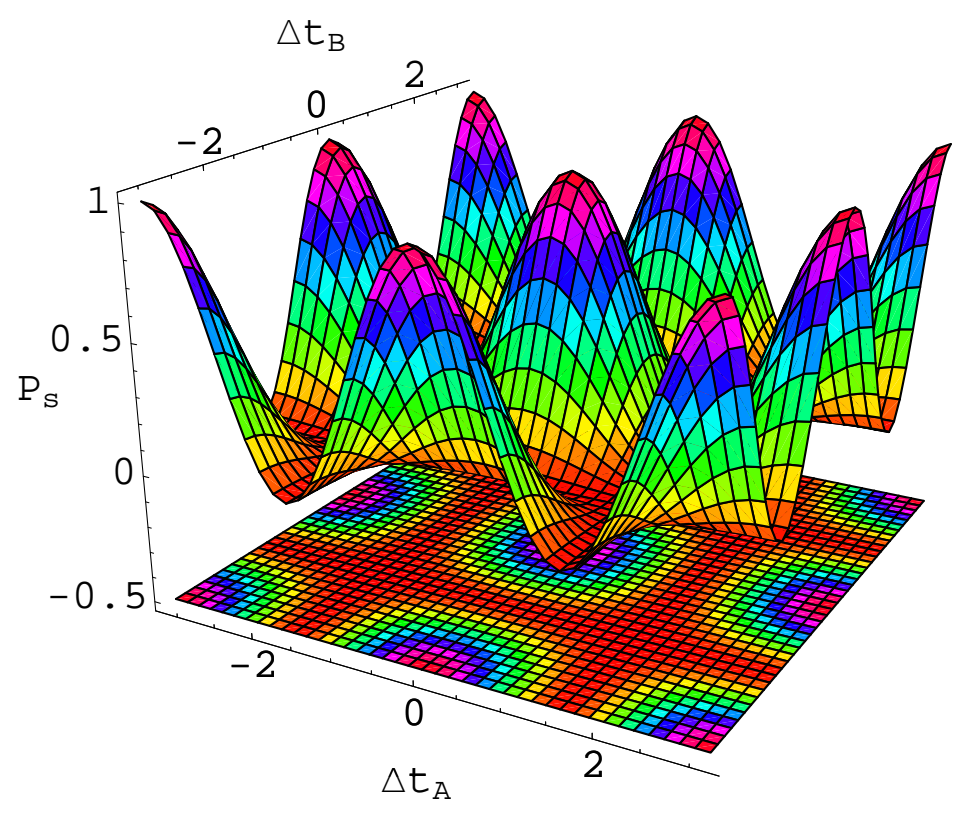

Fig. 2. Success probability $P_{s}$ of a quantum CNOT logic gate versus imprecision in the interaction times $\Delta t_{A}$ and $\Delta t_{B}$ of the atom with field modes $M_{A}$ and $M_{B}$, respectively.

\section{Acknowledgments}

F. S. thanks Prof. S. Watanabe and Prof. G. C. Ghirardi for fruitful discussions. This study was supported by the Quaid-i-Azam University Research Fund and Higher Education Commission under Research Project R\&D03143.

\section{References}

1. R. P. Feynman, Int. J. Theor. Phys., 21, 467 (1982).

2. R. P. Feynman, Opt. News, 11, 11 (1985). 
3. D. Deutsch, Proc. Roy. Soc. (London) A, 400, 97 (1985).

4. M. A. Nielsen and I. L. Chuang, Quantum Computation and Quantum Information, Cambridge University Press, London (2002).

5. Dirk Bouwmeester, Artur Ekert, and Anton Zeilinger, The Physics of Quantum Information, Springer, Berlin (2001).

6. G. Leuchs and T. Beth, Quantum Information Processing, Wiley, Weinheim (2003).

7. I. L. Chuang, L. M. K. Vandersypen, X. Zhou, et al., Nature, 393, 143 (1998).

8. I. L. Chuang, N. Gerschenfeld, and M. Kubinec, Phys. Rev. Lett., 80, 3408 (1998).

9. J. I. Cirac and P. Zoller, Phys. Rev. Lett., 74, 4091 (1995).

10. C. Monroe, D. M. Meekhof, B. E. King, et al., Phys. Rev. Lett., 75, 4714 (1995).

11. J. I. Cirac and P. Zoller, Nature (London), 404, 579 (2000).

12. D. Loss and D. P. Di Vincenzo, Phys. Rev. A, 57, 120 (1998).

13. D. P. Di Vincenzo, Science, 270, 255 (1995).

14. A. Ekert and R. Josza, Rev. Mod. Phys., 68, 733 (1996).

15. J. Preskill, Phys. Today, 52, 24 (1999).

16. A. Khalique and F. Saif, Phys. Lett. A, 314, 37 (2003).

17. R. Islam, M. Ikram, and F. Saif, J. Phys. B: At. Mol. Opt. Phys., 40, 1359 (2007).

18. D. G. Angelakis, M. F. Santos, V. Yannopapas, and A. Ekert, Phys. Lett. A, 362, 377 (2007).

19. Susan M. Clark, Kai-Mei C. Fu, Thaddeus D. Ladd, and Yoshihisa Yamamoto, Phys. Rev. Lett., 99, 040501 (2007).

20. S. Lloyd, Phys. Rev. Lett., 75, 346 (1995).

21. A. Barenco, C. H. Bennett, R. Cleve, et al., Phys. Rev. A, 52, 3457 (1995).

22. D. Deutsch, A. Barenco, and A. Ekert, Proc. Roy. Soc. (London) A, 449, 669 (1995).

23. A. Barenco, D. Deutsch, A. Ekert, and R. Jozsa, Phys. Rev. Lett., 74, 4083 (1995).

24. M. S. Zubairy, M. Kim, and M. O. Scully, Phys. Rev. A, 68, 033820 (2003) and references therein.

25. D.G. Angelakis and A. Kay, ArXiv/quant-ph/0707.3946v1.

26. M. Ikram and F. Saif, Phys. Rev. A, 66, 014304 (2002).

27. M. Ikram and F. Saif, Phys. Rev. A, 67, 069901(E) (2003).

28. A. Rauschenbeutel, P. Berter, S. Osnaghi, et al., Phys. Rev. A 64, 050301(R) (2001).

29. M. O. Scully, and M. S. Zubairy, Quantum Optics, Cambridge University Press (1997).

30. W. P. Schleich, Quantum Optics in Phase Space, Wiley, Berlin (2001).

31. S. Durr, T. Nonn, and G. Rempe, Nature, 395, 33 (1998).

32. S. Kunze, S. Durr, and G. Rempe, Europhys. Lett., 34, 343 (1996). 